\title{
Machine Learning Tackles Spacetime
}

\author{
Neural networks enable an important calculation in a popular approach to unifying quantum \\ mechanics with general relativity.
}

\section{by Enrico Rinaldi ${ }^{1,2}$}

$\mathrm{I}$ $\mathrm{n}$ a breathtaking reveal last year, the Event Horizon Telescope gave the world its first view of a black hole's shadow. But what exactly goes on inside a black hole? General relativity would tell us that a black hole is a singularity in spacetime, a mathematical feature at odds with the fuzziness of quantum mechanics. If scientists want to understand what's happening inside a black hole, they will have to unify the two theories. So far, the most popular formulation of a quantum theory of gravity has been in terms

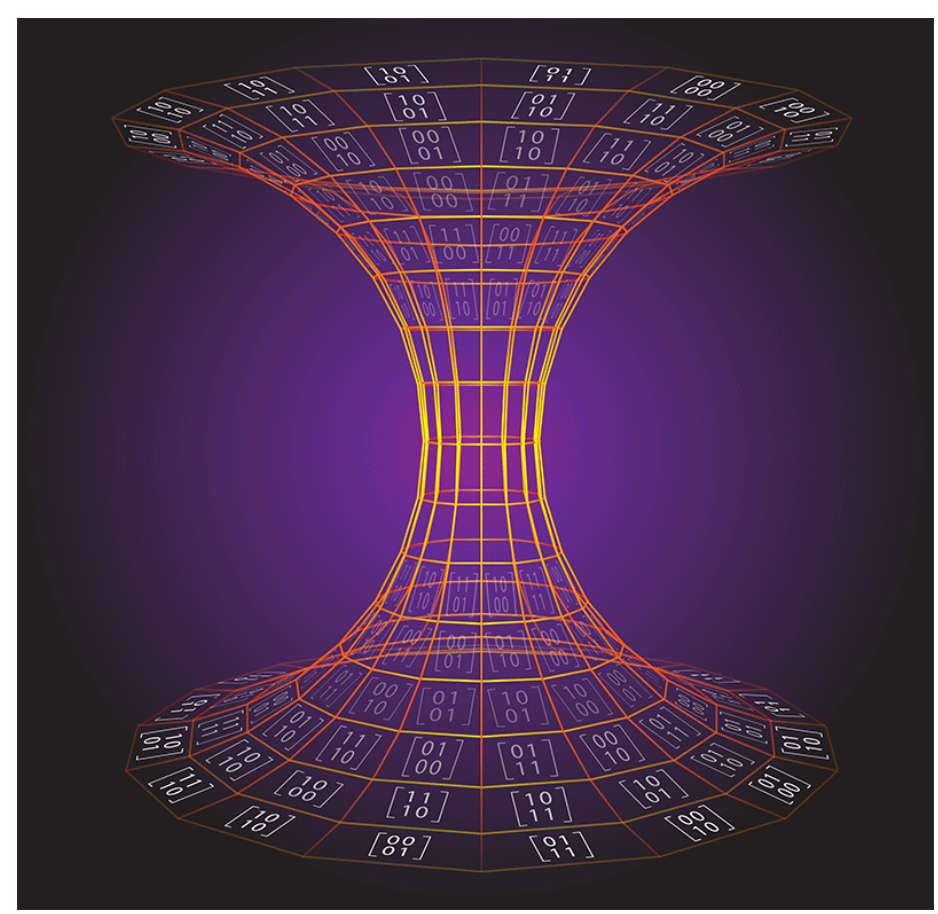

Figure 1: Physicists are taking advantage of neural networks to develop a description of spacetime geometry that is consistent with quantum mechanics. (APS/Alan Stonebraker)

\footnotetext{
${ }^{1}$ Arithmer Inc., Tokyo, Japan

${ }^{2}$ RIKEN Interdisciplinary Theoretical and Mathematical Sciences Program (iTHEMS), Wako, Japan
}

of string theory. A major sticking point, however, has been a prohibitively complex calculation of a quantum-mechanical wave function. New work by Xizhi Han and Sean Hartnoll from Stanford University, California, demonstrates that neural networks - much like those used to generate realistic images of faces-may make this calculation much easier to do [1]. Their results open up a new way to explore the quantum properties of gravity with a computational approach, allowing theorists to "experiment" with gravity.

Unifying general relativity and quantum mechanics into a theory of everything was a dream of Einstein's. Among string theorists, the most promising route to this unification is a conjectured "duality" between certain string theories of gravity and certain quantum (gauge) theories of interacting degrees of freedom (such as particles) [2]. A duality connects two theories that describe seemingly disparate physical systems, much like a dictionary relates the words and concepts of two languages. Physicists find this connection extremely useful because they can solve a very hard problem pertaining to one system in the potentially easier "language" of the other (dual) system. Although the gauge-gravity duality is a conjecture, it has been shown to work for special cases where the same property could be calculated in both the "easy" and "hard" ways [3].

How might the gauge-gravity duality bring us closer to understanding spacetime on a quantum scale? The answer is that the duality allows us to describe the geometry of a black hole-its spacetime shape-in terms of the collective behavior of quantum objects. We can then try to understand how the geometry of spacetime emerges from microscopic degrees of freedom. The wrinkle in this plan is that describing the quantum side of the duality involves prohibitive calculations. String theorists are therefore integrating new computational tools from other disciplines, such as computer science and statistics.

This approach is the spirit behind the new work from Han and Hartnoll, who have used neural networks precisely to describe a system of quantum objects that, though simplified, captures the essential properties of spacetime geometry (Fig. 1). More specifically, they find the ground-state wave function of this many-body system, from which all of the system's properties can be determined from first principles. Calculating such a wave function is notoriously difficult because the wave function is so complex. Moreover, the best method of computing it will usually depend on the wave 
function's mathematical form, which is unknown for the systems relevant to the gauge-gravity duality.

The Stanford duo's approach builds on a pioneering paper from 2016 [4], which showed the potential for finding the many-body wave function using artificial neural networks. Generically, a neural network takes an input, applies a series of mathematical operations to it, and spits out a number. For a lot of familiar applications, a neural network is "trained" with data to recognize inputs (say, a face). In the search for a quantum system's wave function, however, one uses the network's innards to represent a trial wave function and to calculate the system's energy, relying on a separate iteration scheme to choose "better" wave functions that yield a lower energy value.

Taking this approach, Han and Hartnoll adapted a modern neural network to represent the wave function of a quantum system described by the so-called mini-BMN model. This model uses three matrices to represent the system's fundamental degrees of freedom and their interactions [5]. It's a smaller version of the model that's actually dual to the string theoretic description of spacetime near a black hole, which has nine matrices. Researchers have been able to estimate observables associated with mini-BMN using methods such as stochastic Monte Carlo simulations [3]. But these methods are computationally expensive and don't give direct access to the wave function or to geometric properties (though a possible workaround exists [6]). Using the neural network, Han and Hartnoll are able to efficiently extract an approximate wave function with enough information to describe gravity in a previously unexplored quantum regime.

To start, the researchers define the quantum wave function as a parametrized probability distribution over the matrices of the mini-BMN theory. They then use an iterative procedure to find the parameters that minimize the energy of the system. This "training process" is like shaking a bucket of sand until it's level: With each shake, the grains rearrange themselves into a new configuration that removes some of the bumps and lets the sand lie flatter. Similarly, the optimization procedure selects the parameters yielding the "best" ground-state wave function among a family of distributions. Thanks to the fact that they based their neural network on so-called deep generative flows [7], the researchers could efficiently sample many different complex wave functions and find the most accurate one for the ground state.

To benchmark their wave function, Han and Hartnoll use it to calculate certain observables in a semiclassical regime, where their results can be compared to existing calculations. For example, they recover emergent geometric properties predicted in string theory, such as the existence of fuzzy sphere states. They can also describe the quantum entanglement of these states, a key element of quantum gravity.

As noted, the mini-BMN theory describes only a smaller version of the actual quantum system in the gauge-gravity duality. But Han and Hartnoll have ushered in a powerful computational tool for extracting geometrical properties-and that's truly inspiring. This tool can be used as a benchmark for future neural-network algorithms designed for quantum gravity, more of which can be expected now that their potential is clear. Algorithms in computer science have improved at an astonishing rate. If the same progress carries over to the physical sciences [8], a scientific revolution will be afoot. Of course, machine learning is not magic - it works only with certain kinds of inputs and therefore only for certain problems. But it is an important tool that allows scientists to satisfy their curiosity for the unknown. From the confinement of quarks and gluons into protons to the emergence of spacetime, some of the biggest open questions in quantum field theory could benefit from machine-learning tools.

This research is published in Physical Review X.

\section{REFERENCES}

[1] X. Han and S. A. Hartnoll, "Deep quantum geometry of matrices," Phys. Rev. X 10, 011069 (2020).

[2] J. Maldacena, "The Large-N limit of superconformal field theories and supergravity," Int. J. Theo. Phys. 38, 1113 (1999).

[3] E. Berkowitz et al., "Precision lattice test of the gauge/gravity duality at large N," Phys. Rev. D 94, 094501 (2016).

[4] G. Carleo and M. Troyer, "Solving the quantum many-body problem with artificial neural networks," Science 355, 602 (2017).

[5] D. Berenstein et al., "Strings in flat space and pp waves from Script $N=4$ Super Yang Mills," J. High Energy Phys. 4, 013 (2002).

[6] E. Rinaldi et al., "Toward holographic reconstruction of bulk geometry from lattice simulations," J. High Energy Phys. 2, 42 (2018).

[7] G. Papamakarios et al., "Masked autoregressive flow for density estimation," Advances in Neural Information Processing Systems 30 (2017).

[8] G. Carleo et al., "Machine learning and the physical sciences," Rev. Mod. Phys. 91, 045002 (2019).

10.1103/Physics. 13.40 